Full-length article

\title{
Development of a homogeneous calcium mobilization assay for high throughput screening of mas-related gene receptor agonists ${ }^{1}$
}

\author{
Rui ZHANG, Pang-ke YAN, Cai-hong ZHOU, Jia-yu LIAO, Ming-wei WANG² \\ The National Center for Drug Screening, Shanghai Institute of Materia Medica, Chinese Academy of Sciences, Shanghai 201203, China
}

\section{Key words}

mas-related gene receptor; calcium mobilization assay; high-throughput screening

\footnotetext{
${ }^{1}$ Project supported partly by grants from the Ministry of Science and Technology of China (No 2004CB518902) and the Shanghai Municipality Government (No 04XD14023). ${ }^{2}$ Correspondence to Dr Ming-wei WANG Phn 86-21-5080-0598.

Fax 86-21-5080-0721.

E-mail mwwang@mail.shcnc.ac.cn
}

Received 2006-05-19

Accepted 2006-07-31

doi: $10.1111 / \mathrm{j} .1745-7254.2007 .00451 . x$

\begin{abstract}
Aim: To develop homogeneous calcium mobilization assay for high-throughput screening (HTS) of mas-related gene (Mrg) receptor agonists. Methods: CHO-K1 cells stably expressing the full-length MrgD receptor and a calcium-sensitive dye were used to develop an HTS assay based on intracellular calcium influx. This method was applied to large-scale screening of a library containing 8000 synthetic compounds and natural product extracts. cAMP measurements were carried out to verify the bioactivities of the hits found by the calcium mobilization assay. Similar approaches were also employed in the identification of the MrgA1 receptor agonists following HTS of 16000 samples. Results: $\mathrm{EC}_{50}$ values of the positive control compounds ( $\beta$-alanine for MrgD receptor and dynorphin A for MrgA1 receptor) determined by the calcium mobilization assay were consistent with those reported in the literature, and the $Z^{\prime}$ factors were 0.65 and 0.50 for $\mathrm{MrgD}$ and $\mathrm{Mrg} \mathrm{A} 1$ receptor assay, respectively. About 31 compounds for the $\mathrm{MrgD}$ receptor and 48 compounds for the MrgA1 receptor showing $\geq 20 \%$ of the maximal agonist activities found in the controls were initially identified as hits. Secondary screening confirmed that 2 compounds for each receptor possessed specific agonist activities. Intracellular cAMP level measurements indicated that the 2 confirmed hits displayed the functionality of the MrgD receptor agonists. Conclusion: A series of validation studies demonstrated that the homogeneous calcium mobilization assay developed was highly efficient, amenable to automation and a robust tool to screen potential $\mathrm{MrgD}$ and $\mathrm{MrgA} 1$ receptor agonists. Its application may be expanded to other G-protein coupled receptors that mobilize calcium influx upon activation.
\end{abstract}

\section{Introduction}

G-protein coupled receptors (GPCR) constitute one of the largest families of molecular targets applicable to drug discovery in the human genome, and about $40 \%$ to $50 \%$ of currently marketed drugs are directed towards this class of cell surface receptors ${ }^{[1,2]}$. Therefore, they have been the logical choice of numerous high-throughput screening (HTS) efforts to search for new leads of potential therapeutic value.

MrgD and MrgA1 belong to a recently identified family of orphan GPCR genes, namely mas-related genes (Mrg). A putative role for their protein products such as MrgD and MrgA1 receptors in nociception has been postulated based on specific expression in sensory neurons of the dorsal root ganglion (DRG) $)^{[3-5]}$. Studies published in 2004 suggest that they are important regulators in pain perception and conduction, and may provide novel therapeutic opportunities for analgesia ${ }^{[6-8]}$. Although $\beta$-alanine has been shown to stimulate the $\mathrm{MrgD}$ receptor in heterologous systems, the natural ligand remains unknown at this time ${ }^{[9]}$. It was reported that the MrgA1 receptor could be activated by certain opioid-related peptides, but its endogenous ligand(s) is still illusive ${ }^{[3,4,10]}$. Meanwhile, high $\mathrm{EC}_{50}$ values of some known modulators practically limit their application in receptor-binding assays ${ }^{[9]}$. Therefore, new and non-peptide small molecules would serve not only as potential drug leads, but also 
as powerful molecular probes to elucidate the functions of the Mrg receptor family in pain perception.

It is known that both MrgD and MrgA1 receptors are coupled to the G-protein $\alpha$ subunit, Gq. Upon activation, intracellular calcium influx is evoked ${ }^{[9,10]}$, which can be measured using a calcium-sensitive dye and a fluorescence plate reader $^{[11]}$. In the present paper, we describe the development and validation of a homogeneous HTS assay based on the analysis of calcium mobilization using cells expressing $\mathrm{MrgD}$ or MrgA1 receptors. Since the MrgD receptor is also coupled to Gi, confirmed hits were further assessed for their ability to inhibit cAMP content in the MrgD receptor expressing cells.

\section{Materials and methods}

Reagents $\beta$-alanine was purchased from Shanghai Chemical Reagents Co, Ltd (Shanghai, China). FLIPR calcium 3 assay kit and CatchPoint cyclic-AMP fluorescent assay kit were bought from Molecular Devices (Sunnyvale, CA, USA). Dynorphin A (fragment 1-13), probenecid, 3-isobutyl-1methylxanthine (IBMX) and forskolin were obtained from Sigma-Aldrich (St Louis, MO, USA). Fetal bovine serum (FBS) was the product of Hyclone (Logan, UT, USA), cell culture medium F12 and Dulbecco's Modified Eagle's Medium (DMEM) was from GIBCO BRL (Rockville, ML, USA), G418 was from Merck KGaA (Darmstadt, Germany), EcoR I and Xho I were obtained from TaKaRa Biotechnology (Dalian) Co, Ltd (Dalian, China), and Fugene 6 from Roche Diagnostics (Indianapolis, IN, USA).

Plasmid and stable cell lines Full-length MrgD and MrgA1 genes were cloned by PCR using mouse genomic DNA as templates. The PCR primers were as follows:

For the MrgD sense primer:

5'-GGGGAATTCATGAACTCCACTCTTGACAGCAGCCCAGCTCC-3'; anti-sense primer:5'-GGGCTCGAGGACCCCATCATTAGTACACGTGGATGGCGTCTC-3'.

For the MrgA1 sense primer:

5'-GGGGAATTCATGGACAATACCATCCCTGGAGGTATCAACATCAC-3'; anti-senseprimer:5'-GGGCTCGAGTGGCTCTGATTTGCTTCTTGACATCTCCACCATG-3'.

PCR fragments were digested with EcoR I and Xho I, and then cloned into pCMV-Tag2B (Strategene, La Jolla, CA, USA). CHO-K1 (MrgD) or HEK293 (MrgA1) cells (ATCC, Manassas, VA, USA), grown to $50 \%-80 \%$ confluent in a 3.5 $\mathrm{cm}$ culture dish and were transfected with the plasmids using a Fugene $6(\mu \mathrm{L})$ plasmid $(\mu \mathrm{g})$ ratio of 3:1. Four hundred
(MrgA1) or 800 (MrgD) mg/L G418 was added 24h afterwards, and individual clones were selected in 96-well clear culture plates (Corning, Acton, MA, USA) with 400 or $800 \mathrm{mg} / \mathrm{L}$ G418 in DMEM (MrgA1) or F12 medium (MrgD) containing $10 \%$ FBS. Each clone was characterized by the calcium mobilization assay. The stable cells established (MrgD-CHO and MrgA1-HEK293 cells) were maintained in F12 or DMEM medium in the presence of $400 \mathrm{mg} / \mathrm{L} \mathrm{G} 418$.

Calcium mobilization assay MrgD-CHO cells or MrgA1HEK293 cells were detached and plated onto 96-well plates at a density of 30000 cells for MrgD-CHO cells and 20000 cells for MrgA1-HEK293 cells (100 $\mu \mathrm{L} /$ well) and incubated overnight. After changing to $80 \mu \mathrm{L}$ of serum-free medium, the cells were loaded with $80 \mu \mathrm{L}$ of the calcium 3 assay dye supplemented with $2.5 \mathrm{mmol} / \mathrm{L}$ probenecid and incubated at $37^{\circ} \mathrm{C}$ for $60 \mathrm{~min}$. A baseline fluorescence signal was measured for the first $17 \mathrm{~s}$, after which test compounds prepared as a $5 \times$ stock in Hank's balanced salt solution buffer (supplied with the assay kit) were added to the plate through an automated pipetter ( $40 \mu \mathrm{L} /$ well) equipped within FlexStation II $^{384}$ (Molecular Devices, USA). The intracellular calcium influx was analyzed by the same instrument with an excitation wavelength of $485 \mathrm{~nm}$ and emission wavelength of $525 \mathrm{~nm}$, and relative fluorescence signal measured at $1.6 \mathrm{~s}$ intervals for 150-300 s.

cAMP assay MrgD-CHO cells were detached and plated onto 96-well plates at a density of 30000 cells $(100 \mu \mathrm{L} /$ well $)$ and incubated overnight. After 1 wash step with KrebsRinger Bicarbonate Buffer (KRBG) containing $10 \mathrm{mmol} / \mathrm{L}$ glucose, the cells were pre-incubated in $\mathrm{KRBG}$ with $500 \mu \mathrm{mol} / \mathrm{L}$ IBMX for $10 \mathrm{~min}$, and then stimulated with test compounds in the presence of $1.0 \mu \mathrm{mol} / \mathrm{L}$ forskolin for $15 \mathrm{~min}$. Forskolin alone was used as maximal signal control and the blank well as the negative control (background). They were lysed thereafter and quantified for cAMP content using a CatchPoint cyclic-AMP fluorescent assay kit according to manufacturer's instruction. Briefly, $20 \mu \mathrm{L}$ cell lysate or standard cAMP was added to the 384-well plate pre-coated with goat antirabbit IgG, and then $20 \mu \mathrm{L}$ rabbit anti-cAMP antibody followed by $20 \mu \mathrm{L}$ horseradish peroxidase-labeled cAMP. After $2 \mathrm{~h}$ incubation, the plate was washed with $80 \mu \mathrm{L}$ washing buffer 4 times before the addition of $50 \mu \mathrm{L}$ substrate solution. Fluorescence intensity was recorded by FlexStation $\mathrm{II}^{384}$ with an excitation wavelength of $530 \mathrm{~nm}$ and emission wavelength of $590 \mathrm{~nm}$.

HTS campaign The compound libraryused for the screening of MrgD receptor agonists consisted of 8000 pure synthetic compounds and extracts from natural products. A 10compound pool per well mix dissolved in $100 \%$ dimethyl- 
sulphoxide (DMSO) solution was applied to the primary screening, with an average final concentration of $1.7 \mu \mathrm{mol} / \mathrm{L}$ of each compound. This matrix system was known to maximize the advantage of HTS and allowed duplicate screening of each compound ${ }^{[12]}$. In each of the 96-well plate, 16 wells were used either as the positive control $(\beta$-alanine or dynorphin A) or as the negative control (assay buffer), and samples showing greater than $20 \%$ agonist activities compared to the maximum response elicited by the positive control were considered 'hits'.

Data analysis Data were analyzed using GraphPad Prism software (San Diego, CA, USA). Non-linear regression analyses were performed to generate dose-response curves to calculate $\mathrm{EC}_{50}$ values. Time-course study results from the calcium mobilization assay were expressed as relative fluorescence unit (RFU), and all other calcium influx measurements were represented as the peak RFU per well. Values presented are mean \pm SEM of at least 3 independent experiments. Percentage responses of hits were estimated based on the response to $1.0 \mathrm{mmol} / \mathrm{L} \beta$-alanine or $60 \mu \mathrm{mol} / \mathrm{L}$ dynorphin A, which was defined as $100 \%$. Z' factors were calculated as described by Zhang et $a l^{[13]}$.

\section{Results}

Assay validation In the present study, we first assessed the kinetics of the signal generated in the assay system. Time-course experiments showed that agonist stimulation with $1.0 \mathrm{mmol} / \mathrm{L} \beta$-alanine produced a significant increase in fluorescence intensity relative to the background (non $\beta$ alanine stimulated) and the peak RFU appeared around $70 \mathrm{~s}$ followed by a gradual signal decay to the baseline (Figure 1A). As for the MrgA1 receptor, a similar time-course curve was observed with $60 \mu \mathrm{mol} / \mathrm{L}$ dynorphin A (Figure 1B). Meanwhile, $\beta$-alanine and dynorphin $A$ each did not induce any increase in fluorescence intensity in the blank $\mathrm{CHO}$ or HEK293 cells (data not shown). The effects of cell seeding density on the assay signal and background are shown in Figure 2. A density of 30000 cells per well for MrgD-CHO and 20000 cells per well for MrgA1-HEK293 generated the best signal to background (S/B) ratios (58.1 and 50.3). These numbers were selected for respective HTS campaign. DMSO, at concentrations of up to $0.5 \%$, did not affect the assay performance (data not shown). Under these optimized conditions, the determined $\mathrm{EC}_{50}$ values of the known $\mathrm{MrgD}$ receptor ligand $\beta$-alanine and the MrgA1 receptor agonist dynorphin A were $8.6 \mu \mathrm{mol} / \mathrm{L}$ and $1.1 \mu \mathrm{mol} / \mathrm{L}$, respectively (Figures 3A, 3B), consistent with those reported in the literature $^{[3,9]}$.

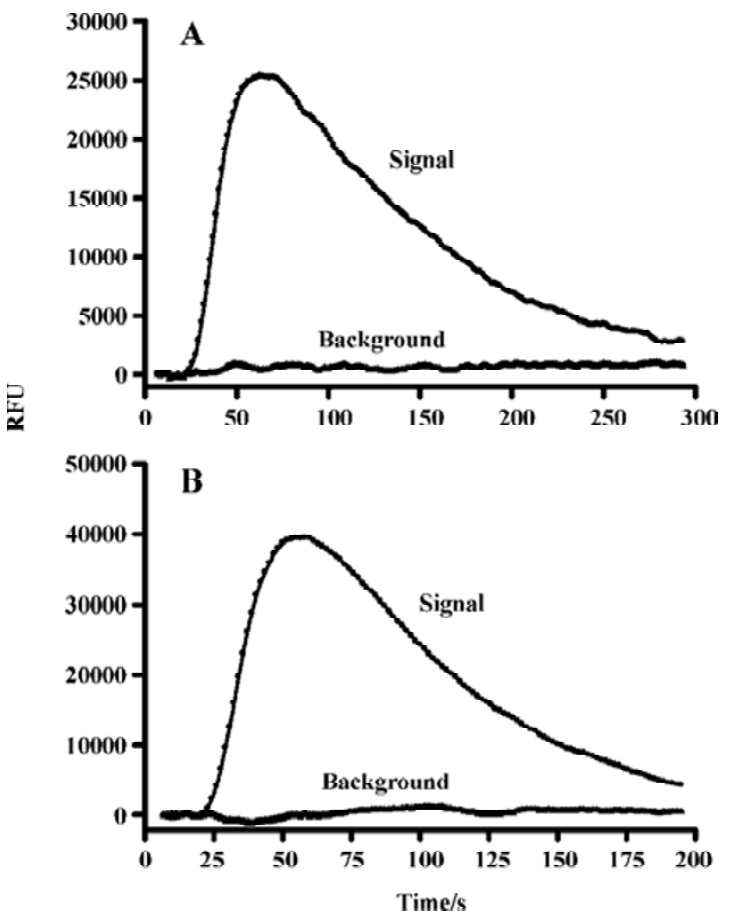

Figure 1. Kinetics curves of $1.0 \mathrm{mmol} / \mathrm{L} \beta$-alanine (A) and 60 $\mu \mathrm{mol} / \mathrm{L}$ dynorphin A (B) that evoked an increase of intracellular calcium concentration, monitored by changes in fluorescence units in MrgD-CHO (A) and MrgA1-HEK293 (B) cells. The background is a record of cell response where $\beta$-alanine or dynorphin A was absent. Data shown are representative traces.

Assay performance In order to apply this assay system to HTS format, both signal intensity evoked by $1.0 \mathrm{mmol} / \mathrm{L}$ $\beta$-alanine and background noise without alanine were extensively studied. As shown in Figure 4, the $Z^{\prime}$ value for the calcium mobilization assay was 0.65 with an $\mathrm{S} / \mathrm{B}$ ratio of 54.0, and the coefficient of variation $(\mathrm{CV})$ value for the signal was $10.7 \%$. In the case of the MrgA1 receptor, the $Z$ factor, S/B ratio, and $\mathrm{CV}$ value for the signal were $0.5 \%, 73.6 \%$, and $15.8 \%$, respectively (Figure 4B). These parameters indicate that the system was adequately optimized for HTS.

Identification of MrgD receptor agonists Of the 8000 samples initially screened for potential MrgD receptor agonists, 31 initial hits $(0.39 \%)$ showing greater than $20 \%$ agonistic activities relative to $\beta$-alanine were discovered, as shown in the scatter plot of the actual readouts (Figure 5). The HTS campaign resulted in a $Z^{\prime}$ value of 0.6 that conferred the assay quality requirement of above $0.5^{[13]}$. Secondary screening with a single compound per well confirmed that 14 of the above 31 hits displayed consistent agonistic properties. Follow-up experiments using CHO cells lacking the MrgD receptor revealed that only 2 of the above 14 hits 

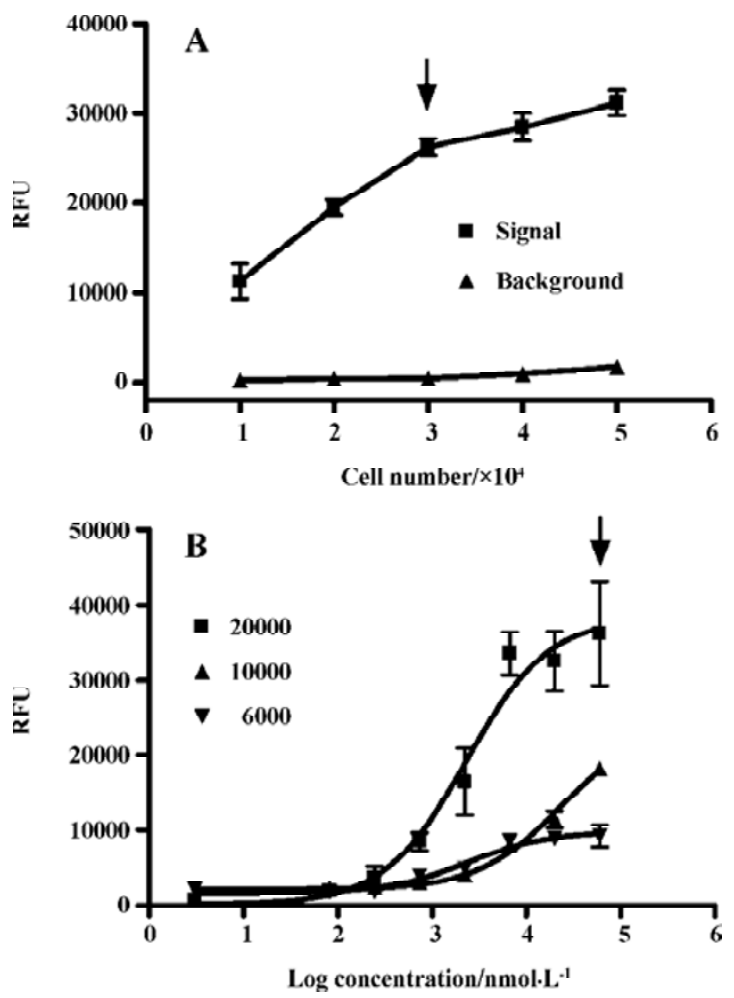

Figure 2. Effects of different cell densities on the calcium mobilization signal and background read-out in MrgD-CHO (A) or MrgA1HEK293 (B) cells in the presence and absence of $1.0 \mathrm{mmol} / \mathrm{L} \beta$ alanine (A) or different concentration of dynorphin A (B). Data are mean \pm SEM. $n \geq 3$. Arrows indicate the cell density selected for HTS.
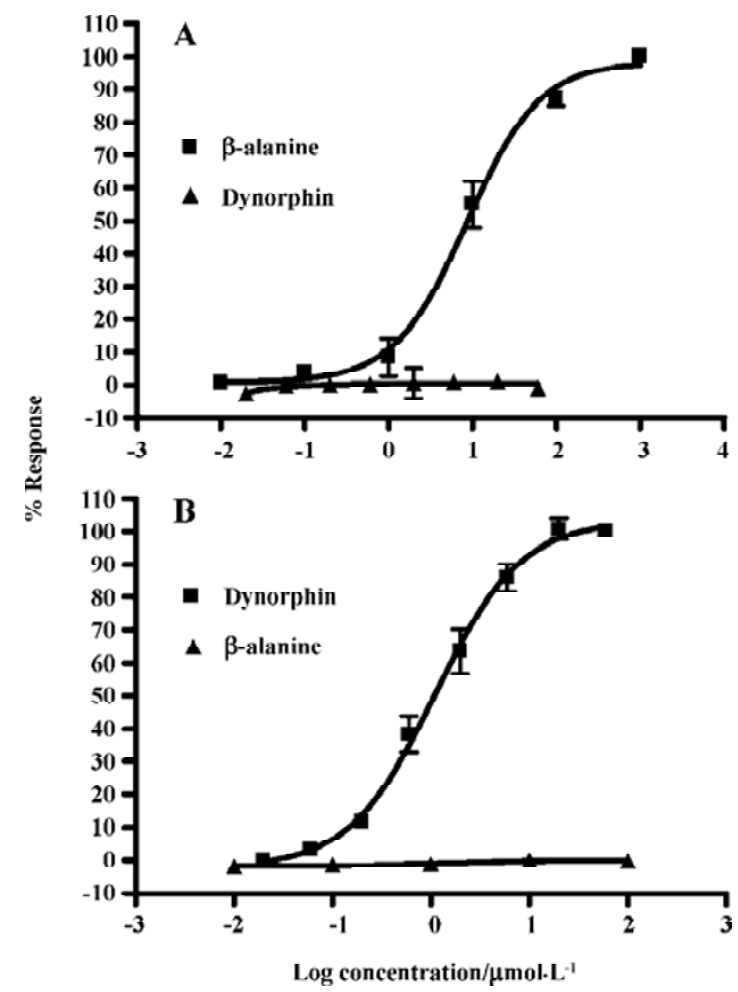

Figure 3. Dose-response curves of $\beta$-alanine and dynorphin $\mathrm{A}$ in MrgD-CHO (A) and MrgA1-HEK293 (B) cells measured by the calcium mobilization assay from which $\mathrm{EC}_{50}$ values were calculated. Data are mean \pm SEM. $n \geq 3$.

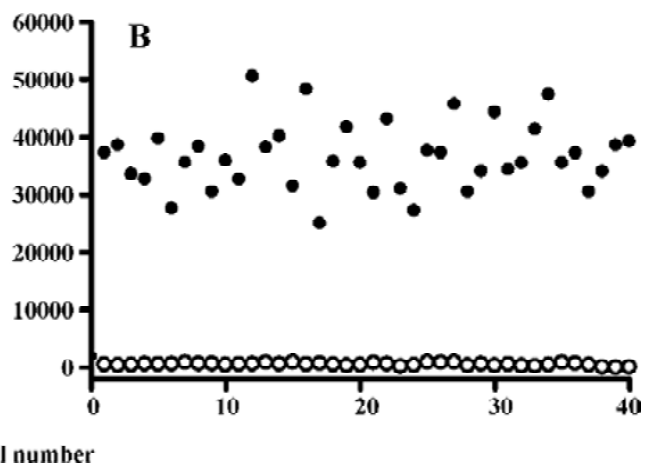

Figure 4. Z' factor determination. Calcium mobilization assay was performed at optimized conditions in MrgD-CHO (A) or MrgA1-HEK293 (B) cells stimulated with $1.0 \mathrm{mmol} / \mathrm{L} \beta$-alanine (A) or $60 \mu \mathrm{mol} / \mathrm{L}$ dynorphin $\mathrm{A}$ (B). The background is a record of cell response where $\beta$-alanine or dynorphin A was absent. Forty replicates of signal and background read-outs were studied.

elicited calcium influx through an MrgD receptor-mediated mechanism. They were further studied in a functional assay where intracellular cAMP levels were measured following $\mathrm{MrgD}$ activation. When validating the assay, $\beta$-alanine did not exhibit any effect on the cAMP levels in the blank $\mathrm{CHO}$ cells (data not shown). As displayed in Figure 6, like $\beta$ alanine, both hits (121145 and 011531) induced calcium influxes and cAMP responses in CHO cells expressing MrgD, thus verifying their agonist status.

Identification of MrgA1 receptor agonists Based on the 


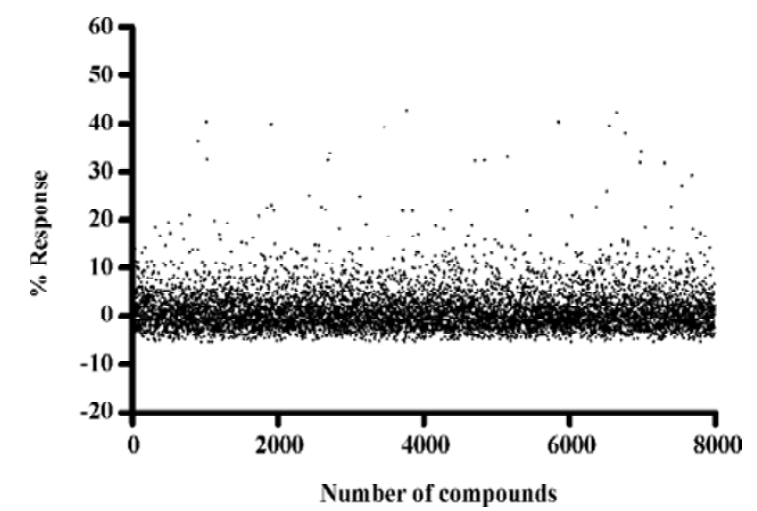

Figure 5. High-throughput screening of 8000 compounds using the calcium mobilization assay in MrgD-CHO cells. Results are expressed as percentage response relative to $1.0 \mathrm{mmol} / \mathrm{L} \beta$-alanine $(100 \%)$.
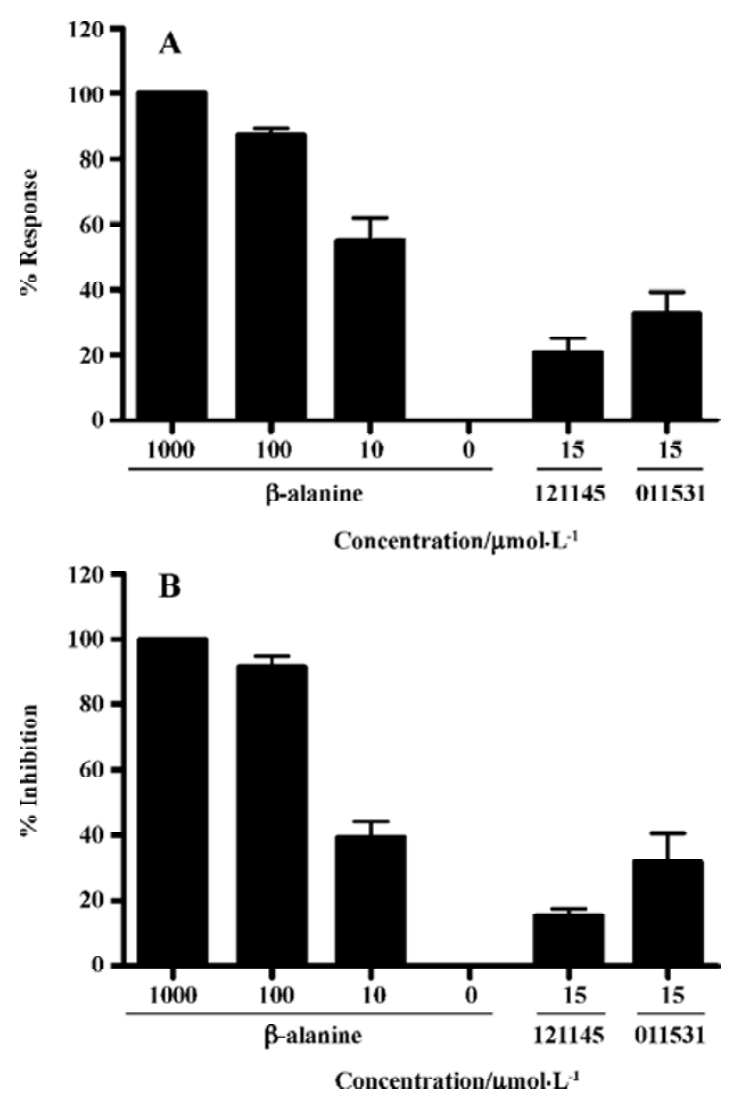

Figure 6. Effects of 2 synthetic small molecule hits (121145 and 011531 ) on intracellular calcium influx (A) and cAMP levels (B) in MrgD-CHO cells. Results are expressed as percentage response (A) or inhibition (B) relative to $1.0 \mathrm{mmol} / \mathrm{L} \beta$-alanine $(100 \%)$. Data are mean \pm SEM. $n \geq 3$.

above HTS experience, we applied the same method to screen MrgA1 receptor agonists. Briefly, HEK293 cells expressing $\mathrm{MrgA} 1$ were used to optimize assay conditions. As a result, a cell density of 20000 per well and DMSO tolerance of $0.5 \%$ were selected for the HTS campaign. The assay parameters studied were comparable to that of MrgD (Table 1), suggesting its suitability. Following a major HTS campaign on 16000 synthetic compounds or natural product extracts, 48 initial hits $(0.30 \%)$ showing greater than $20 \%$ agonistic activities relative to dynorphin A were discovered (Figure 7). Follow-up screening with blank HEK293 cells confirmed that 2 hits (077802 and 078728) consistently displayed MrgA1 agonist effects on calcium mobilization (Figure 8). These, together with the $2 \mathrm{MrgD}$ agonists above, were further examined by reciprocal calcium mobilization assay to check their specificities for $\mathrm{MrgD} v s \mathrm{MrgA} 1$ receptors. No crossreactivity was noted between the 2 sets of hits (Table 2).

Table 1. HTS assay parameters.

\begin{tabular}{lll}
\hline \multicolumn{1}{c}{ Parameter } & MrgD receptor & MrgA1 receptor \\
\hline Samples screened & 8000 & 16000 \\
Z' value & 0.65 & 0.50 \\
\% Primary hits & 0.39 & 0.30 \\
Confirmed hits & 2 & 2 \\
\hline
\end{tabular}

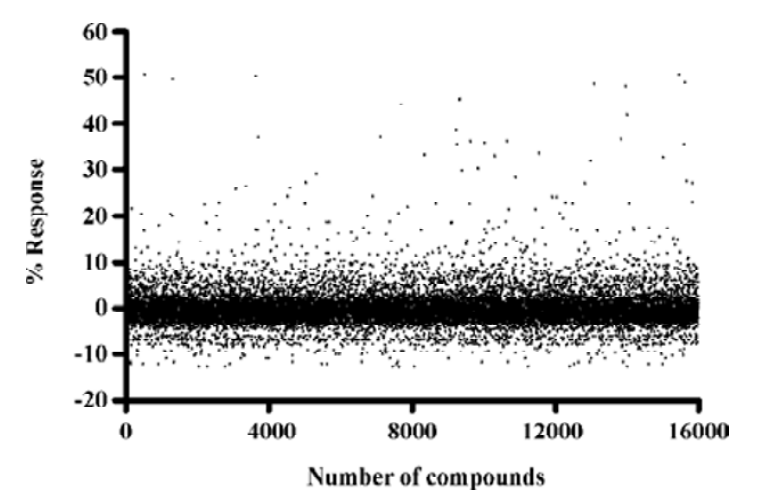

Figure 7. High-throughput screening of 16000 compounds using the calcium mobilization assay in MrgA1-HEK293 cells. Results are expressed as percentage response relative to $60 \mu \mathrm{mol} / \mathrm{L}$ dynorphin A $(100 \%)$.

\section{Discussion}

Opioids have been used clinically as effective analgesics for many pain conditions, but their application is limited by considerable central nervous system (CNS) mediated sideeffects. Therefore, the development of peripheral analgesics that do not cross-react with central opioid receptors is of common interest for many researcher ${ }^{[16]}$. The restricted expression of $\mathrm{MrgD}$ and $\mathrm{MrgA1}$ in sensory neurons of $\mathrm{DRG}$ 


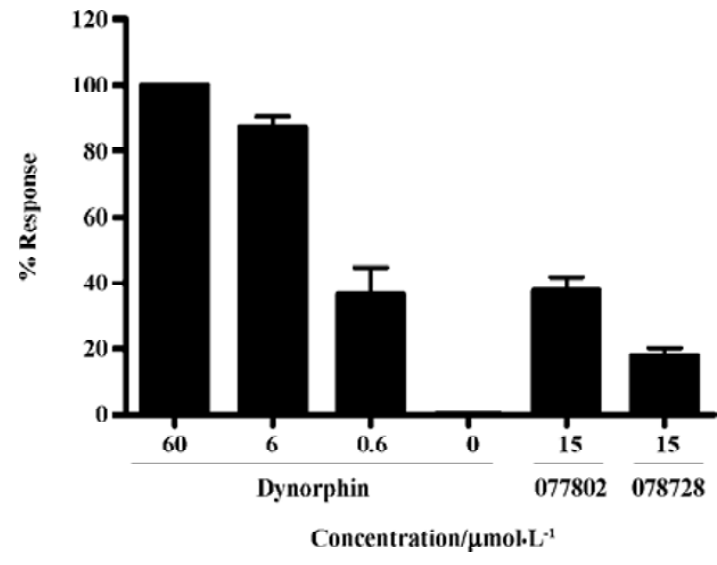

Figure 8. Effects of 2 synthetic small molecule hits (077802 and 078728) on intracellular calcium influx in MrgA1-HEK293 cells. Data are mean \pm SEM. $n \geq 3$.

Table 2. Cross-activity of confirmed hits for MrgD and MrgA1 receptors in calcium mobilization assay.

\begin{tabular}{llcc}
\hline Compound & Structural class & $\begin{array}{c}\text { MrgD } \\
\text { receptor }\end{array}$ & $\begin{array}{c}\text { MrgA1 } \\
\text { receptor }\end{array}$ \\
\hline -alanine & Amino acid & + & - \\
121145 & Puridazine & + & - \\
011531 & Thiazole & + & - \\
Dynorphin A & Peptide & - & + \\
077802 & Quinoline & - & + \\
078728 & Imine & - & + \\
\hline
\end{tabular}

+ , response that is equal or more than $20 \%$ of the control; -, response that is below $1 \%$ of the control.

offers a new strategy in the study of peripherally active analgesics to mitigate or avoid undesirable CNS side-effects such as mental impairment and drug dependence. This is the rationale behind the studies presented in this paper.

Cell-based assays can offer a wealth of information relative to the functionality of a test compound, and thus are widely employed in the drug discovery process. As far as measuring intracellular calcium influx is concerned, cells are loaded with the acetoxymethyl ester of Fluo-3 or Fluo-4 in a typical assay format, and unincorporated dye is removed by repeated washing. Obviously, this non-homogeneous approach is not sufficiently robust for HTS. Fluorometric imaging plate reader (FLIPR) calcium 3 is a newly developed fluorescent dye (calcium indicator) that uses quenching technology to reduce background fluorescence, thereby providing a homogenous solution ${ }^{[14]}$. A major practical benefit of this method is that it eliminates cell-washing steps, resulting in easier automation and faster throughput. As demonstrated in the present study, compounds were screened at a rate of 20 s per well; a minimum of 4000 compounds could be screened each day in a 96-well plate format ( 1 compound per well). This advantage could be further explored by utilizing highdensity plates (eg 384-well plate).

As Mrg receptors are still classified as orphan GPCR, their implication as potential drug targets requires vigorous validation. Little knowledge exists today regarding the native ligands, which makes assay development extremely difficult. Apart from discovering relevant modulators to alleviate pain symptoms, confirmed hits may well serve as molecular probes to the understanding of Mrg receptor functions. Conceivably, exogenous ligands would be more easily screened in a format in which the target is expressed and regulated in the most physiologically relevant manner. When an artificial system such as an engineered cell line is employed, many factors must be considered in order to maximize the $\mathrm{S} / \mathrm{B}$ ratio. As shown in Figure 2, varying cell seeding density influences the signal and background of the cellular reaction. We chose 20000 or 30000 cells/well in the HTS setting that resulted in an optimal $\mathrm{S} / \mathrm{B}$ ratio. In addition, DMSO tolerance can limit the maximum concentration of the compound to be assayed ${ }^{[15]}$. In our experience, $0.5 \%$ DMSO did not disturb the assay performance and a final compound concentration of $17 \mu \mathrm{mol} / \mathrm{L}$ was achieved.

The Z' factor is a useful tool for evaluating bioassay qualities. In general, a $Z^{\prime}$ value above 0.5 suggests that an assay is robust enough for HTS. The calcium mobilization system described herein consistently displayed a $Z^{\prime}$ value between 0.50 and 0.65 . This, and in conjunction with other parameters, such as $\mathrm{S} / \mathrm{B}$ ratio as well as $\mathrm{CV}$ values, indicates that the assay is of a high quality nature.

False positives are a major problem for any type of HTS assay. In the calcium mobilization analysis, this translates into issues such as auto-fluorescence of test compounds, calcium ionophores and chemicals that permeabilize the cell membrane ${ }^{[11]}$. In our case, the majority of the non-specific compounds were discarded after running a secondary screening using cells lacking MrgD or MrgA1 receptors. The bioactivities of the confirmed hits for MrgD discovered in the calcium mobilization assay were supported by a decrease in the intracellular cAMP level, an indicator of Gi activation, and strikingly similar potencies determined by these 2 different functional assays.

Because the MrgA1 receptor is only coupled to Gq, but not to the Gi or Gs signaling pathway ${ }^{[10]}$, cAMP measurements are not applicable in terms of functionality verification. 
Since the homology in DNA sequences of the 2 Mrg receptor genes is only $37.9 \%{ }^{[3-5]}$, it is not surprising that the hits for the MrgD receptor did not cross-react with the MrgA1 receptor or vice versa. This feature is of importance in the development of subtype-selective Mrg receptor modulators for therapeutic use. In addition, the structural diversity of the 4 confirmed hits (Table 2) will certainly offer a good starting point for medicinal chemistry efforts as well as an indepth structure-activity relationship exploration.

In summary, we have successfully developed and evaluated a homogeneous calcium mobilization assay that can be applied to HTS for small molecule MrgD or MrgA1 receptor agonists. The hits identified by the system could be functionally verified by another assay based on a known signal transduction pathway. This approach is straightforward, robust and amenable to automation, with a minimum screening throughput of 4000 compounds per day. It may also be applied to other GPCR that involve calcium signaling upon stimulation.

\section{Acknowledgements}

The authors are indebted to Dr Xin XIE (The National Center for Drug Screening, Shanghai Institute of Materia Medica, Chinese Academy of Sciences, Shanghai) for technical advice and to Dr Dale E MAIS (Ligand Pharmaceuticals, Inc, USA) for critical review of the manuscript.

\section{References}

1 Hopkins AL, Groom CR. The druggable genome. Nat Rev Drug Discov 2002; 1: 727-30.

2 Wise A, Gearing K, Rees S. Target validation of G-protein coupled receptors. Drug Discov Today 2002; 7: 235-46.

3 Dong X, Han S, Zylka MJ, Simon MI, Anderson DJ. A diverse family of GPCRs expressed in specific subsets of nociceptive sensory neurons. Cell 2001; 106: 619-32.

4 Lembo PM, Grazzini E, Groblewski T, O’Donnell D, Roy MO,
Zhang J, et al. Proenkephalin A gene products activate a new family of sensory neuron-specific GPCRs. Nat Neurosci 2002; 5: 201-9.

5 Zylka MJ, Dong X, Southwell AL, Anderson DJ. Atypical expansion in mice of the sensory neuron-specific Mrg G protein-coupled receptor family. Proc Natl Acad Sci USA 2003; 100: 10043-8.

6 Grazzini E, Puma C, Roy MO, Yu XH, O’Donnell D, Schmidt R, et al. Sensory neuron-specific receptor activation elicits central and peripheral nociceptive effects in rats. Proc Natl Acad Sci USA 2004; 101: 7175-80.

7 Hong Y, Dai P, Jiang J, Zeng X. Dual effects of intrathecal BAM22 on nociceptive responses in acute and persistent painpotential function of a novel receptor. Br J Pharmacol 2004; 141: 423-30.

8 Chen H, Ikeda SR. Modulation of ion channels and synaptic transmission by a human sensory neuron-specific G-proteincoupled receptor, SNSR $4 / \mathrm{mrgX} 1$, heterologously expressed in cultured rat neurons. J Neurosci 2004; 24: 5044-53.

9 Shinohara T, Harada M, Ogi K, Maruyama M, Fujii R, Tanaka H, et al. Identification of a $\mathrm{G}$ protein-coupled receptor specifically responsive to beta-alanine. J Biol Chem 2004; 279: 23559-64.

10 Han SK, Dong X, Hwang JI, Zylka MJ, Anderson DJ, Simon MI. Orphan G protein-coupled receptors MrgA1 and $\mathrm{MrgC} 11$ are distinctively activated by RF-amide-related peptides through the Galpha q/11 pathway. Proc Natl Acad Sci USA 2002; 99: 14740-5.

11 Johnston PA, Johnston PA. Cellular platforms for HTS: three case studies. Drug Discov Today 2002; 7: 353-63.

12 Hann M, Hudson B, Lewell X, Lifely R, Miller L, Ramsden N. Strategic pooling of compounds for high-throughput screening. J Chem Inf Comput Sci 1999; 39: 897-902.

13 Zhang JH, Chung TD, Oldenburg KR. A simple statistical parameter for use in evaluation and validation of high throughput screening assays. J Biomol Screen 1999; 4: 67-73.

14 Zhang Y, Kowal D, Kramer A, Dunlop J. Evaluation of FLIPR calcium 3 assay kit-a new no-wash fluorescence calcium indicator reagent. J Biomol Screen 2003; 8: 571-7.

15 Moore K, Rees S. Cell-based versus isolated target screening: how lucky do you feel? J Biomol Screen 2001; 6: 69-74.

16 Feinberg SD. Prescribing analgesics. How to improve function and avoid toxicity when treating chronic pain. Geriatrics 2000; 55: 44-53. 